Brit. F. vener. Dis. (1967), 43, 128.

\title{
AN ANALYSIS OF SOME CHARACTERISTICS OF MALES WITH GONORRHOEA*
}

\author{
BY \\ L. H. GLASS \\ Department of Health Science, San Fernando Valley State College, Northridge, California
}

Much of the research in the field of gonorrhoea causation has been restricted to the diagnostic, epidemiological, and therapeutic aspects of gonorrhoea control. Few efforts have been made to study scientifically the human and social elements which appear to play significant roles in the problem. In these latter areas, fact has often given way to impression, and too often traditions have dominated. The majority of books, papers, and articles published in the field of gonorrhoea control have less to do with the social aspects of venereal disease than with the factors already outlined.

In Los Angeles, as in many parts of the United States, the reported incidence of gonorrhoea has been increasing for several years. The increase has occurred despite the efficiency of drug therapy, increased case finding, and well-equipped and well-staffed public health clinics. Existing gonorrhoea control programmes are inadequate, primarily because they do not consider the whole patient. For years, the basic principles of a gonorrhoea-control programme included the identification of infectious individuals as quickly as possible; the location of the source of the infection; the identification and examination of the sex contacts with the primary source; and the provision of adequate therapy or prophylaxis. While such an approach is medically adequate for the treatment of infection, it fails to prevent re-infection because the social and psychological aspects affecting the patient's behaviour are not considered. When programmes of various agencies involved in gonorrhoea control are examined, it becomes obvious that the major emphases are still being placed upon these same basic principles. While these efforts are extremely important, it is difficult to see how gonorrhoea can be eradicated or effectively reduced in incidence as long as this

\footnotetext{
*Received for publication August 31, 1966.
}

approach remains the primary focus of attention. Ths history of public health reveals that diseases such as smallpox, cholera, typhoid fever, poliomyelitis, and diphtheria, to name a few, were not eliminated by measures of early diagnosis and treatment. Rather, they were effectively reduced by measures of prevention that interrupted the chain of events involving man. These measures included vaccination, immunization, and environmental health procedures. In the light of this historical background, the same question may be asked in relation to venereal disease in general and gonorrhoea in particular. Can these problems be effectively reduced if the attack is concentrated on case finding? If the same principles operate as for the examples quoted, the answer would appear to be no. As a result, it is necessary to turn to additional means of prevention. One possible approach is to continue the search for immunization measures. Another approach is through the application of health education as a primary measure of prevention.

For health education to be feasible in the reduction of gonorrhoea, it can no longer be thought of purely as a medical entity. Gonorrhoea is not usually acquired by man accidentally. It is the result of a deliberate act. As such, it can be regarded as a symptom of the behaviour which was responsible for the act. The complex factors that are involved in the acquisition and transmission of the disease have their origins in variations of human behaviour, in sexual promiscuity, and perhaps in some of the inadequacies of social and economic life.

If individuals are to be prevented from acquiring gonorrhoea and repeated re-infections, it is necessary for a change in behaviour to occur. It is in this area that health education can be of assistance. Contemporary health education is concerned with the behaviour of individuals and groups. How a 
person thinks and acts in specific situations seems to depend upon a number of variables-motivational, perceptual, social, and cultural. This concept emphasizes the need for the health educator (or those concerned with planning educational programmes) to obtain far more specific information about individuals of a particular group as essential background information. This information can assist the health educator in evaluating decisionmaking for health action by individuals in these groups. On this basis, he can select more effective health education approaches that may provide better motivation for a desired course of action, i.e. decreased risk of infection with gonorrhoea.

One of the major problems in the field of venereal disease control is the male patient with gonorrhoea who has had a previous attack of the disease and whose practices have led to repeat infections. Current public health procedures have failed significantly to reduce this problem. Various public health investigators have reported that well over 50 per cent. of the males treated for gonorrhoea have had a previous infection during their lifetime. Chapman (1958), in an unpublished paper, "Penicillin Prophylaxis in Prevention of Gonorrheal Reinfection" stated:

"In one venereal disease clinic in Los Angeles, staff members estimate that up to 85 percent of males treated for acute gonorrhea have had a prior attack of the disease. In March, 1954, for example, 209 males were admitted there with gonorrhea. Almost 65 percent (133 of 209) reported one or more prior attacks of gonorrhea during the 12 months preceding treatment. Of these 133 patients, nearly half succumbed to reinfection within thirty days following treatment."

\section{Los Angeles Study}

The aim of this study was to gain additional knowledge and insight into the social and psychological factors of individuals who have or suspect they have gonorrhoea in order to obtain improved clues for prevention of re-infection. Basic to an understanding of the problem of such individuals and the potentialities for health education is the necessity to identify the behavioural characteristics.

The study universe was limited to every third Negro male who appeared for the diagnosis and treatment of gonorrhoea at the Special Medicine Clinic of the Southeast Health District, Los Angeles City Health Department, a total of 150 males. This particular clinic is the largest in terms of VD patients in the Los Angeles area. In addition, the clinic deals with many individuals who have re-infections of gonorrhoea. Most of the clinic patients are residents of the district.
An interview schedule was developed utilizing the patient's knowledge of gonorrhoea and related behaviour, social characteristics, and personality characteristics. Certain questions relating to social and personality characteristics were adapted from two studies of similar socio-economic groups: Reeder's Los Angeles Maternity Survey (1961) and the Johns Hopkins Longitudinal Study of Wilner, Walkley, Pinkerton, and Tayback (1962).

The final instrument consisted of 172 questions designed to provide basic information on a number of characteristics:

(a) Demographic Items Personal characteristics, including age, race, marital status, number of times married, rural-urban background, religious background and present affiliation, education and occupational status (Reeder, 1961).

(b) Social Class Position Including social class position of respondent, mother or father (Reeder, 1961).

(c) Residential Characteristics Including length of time in California and Los Angeles, location of previous residence, length of time at present address, index of trend of residential mobility, number of places lived in during last five years, "necessity moves" and "choice moves", monthly rent, and room-person ratio.

(d) Social Participation Including participation in organized social groups or activities, the number of close friends, in their neighbourhoods and their comparative ages, and the frequency of visiting these friends (Reeder, 1961).

(e) Stressful Living Conditions Involving such personality characteristics as mood, temper, nervousness and spontaneous expression (Wilner and others, 1962).

(f) General Morale Involving such personality characteristics as self-help, status quo and optimismpessimism (Wilner and others, 1962).

(g) Strictness of Obedience Involving opposition to authority as a personality characteristic (Wilner and others, 1962).

(h) Social Self-confidence Involving such personality characteristics as self-esteem and self-confidence (U.C.L.A., 1963).

(i) Previous History of Gonorrhoea Including number of previous infections, age at first infection, where treatment was sought, and how soon.

(j) Knowledge about Gonorrhoea Including the cause, treatment, effects, and methods of prevention (Chapman, 1958).

For the purposes of administering the questionnaire, the interviewer selected every third male who was in the clinic because of problems relating to gonorrhoea. Since the registrants had not been 
examined by the clinician, it was possible for individuals who were not infected to be interviewed. These individuals were also included in the survey. The interviewing was done at the time of the diagnostic and treatment visit in a separate office in the clinic area in order to ensure privacy and confidentiality.

In order to minimize interviewer variation, all interviews were administered by the investigator. All the questions were read to the respondent and the answers recorded upon the questionnaire. Each questionnaire was identified by the patient's name and case record number.

The time needed to complete the interview instrument ranged from 30 to 45 minutes. Each patient was told at the beginning of the interview that a research project was being undertaken to find out more about people who have gonorrhoea. The ultimate purpose of the research was explained as being an attempt to gain more knowledge to help those who become infected with gonorrhoea. Each patient was told that his participation was strictly voluntary. He could refuse to answer any or all questions. The patients were extremely cooperative, and only a few declined to answer certain questions.

Data related to the number of previous episodes treated at this same clinic, marital status, occupation, age, and number of children were available in clinic charts and could have been utilized to validate the responses provided by the patient. While these data were referred to, they were not recorded.

\section{Analyses}

The investigation for the study centred in two major aspects, namely: to obtain information regarding behavioural and other characteristics of individuals who have or suspect they have gonorrhoea; to examine data in such a way as to identify characteristics related to the number of infections of gonorrhoea.

Preliminary analysis of the data revealed that a number of the factors were providing the same kind of information. As a result, the factors were carefully screened to exclude those that fell into this category; 33 were selected for further analysis of possible relationships with the number of infections in a lifetime, a year, and 3 months.

From the analyses, it appeared that age and age-related factors seemed to be relatively important when the 33 factors were compared individually with the number of infections in a lifetime. As a result, the question was raised: "Is age the only important variate; does it distinguish between those who acquire multiple infections as opposed to those who do not ?". In order to examine this observation more closely, selected factors were compared against the actual number of infections in a lifetime, a year, and 3 months (instead of the arbitrary classification of $0,1,2,3$, and more than 3) in two-way tables. In this way, the number of persons in the study distributed themselves according to the number of actual infections. The variates showing the highest significance in the $\chi^{2}$ test were then used in further comparisons. From this analysis, the factors, age at first infection, self-help, and opposition to authority were selected.

When age at first infection was adjusted for the factors self-help and opposition to authority respectively, it failed to show an obvious relationship with the number of infections. One might postulate that the age factor may be modified by other factors and may not be the important factor related to fewer or more infections. This suggests that health education programmes may not be effective if based upon the consideration of these factors, one or even two at a time. Behaviour leading to infections of gonorrhoea is too complex to measure on this basis.

The multiple linear regression model (a method of multifactorial analysis) was utilized as a method of considering all the variates simultaneously in an effort to establish a relationship between number of infections and social characteristics, knowledge of gonorrhoea, and related behaviour and personality characteristics. This analysis suggested that these characteristics, considered simultaneously, do bear some relationship to the number of infections in a lifetime, but not so strong a relationship to infections in a year or in 3 months.

Because the study dealt with people having a random length of time to acquire a random number of infections, and since age at first infection and present age had the highest and third highest regression coefficients respectively, the analysis was then oriented to investigate the following question: If everyone had the same amount of time in which to acquire infections (on the average), would the characteristics in the interview schedule be related to the number of infections acquired? The analysis suggested that, even with time factors held constant in this way, social characteristics, knowledge of gonorrhoea, and related behaviour and personality factors considered simultaneously seem to bear some relationship to the total number of infections at interview.

The next point of inquiry dealt with reducing the number of interview characteristics used in 
establishing the observed relationship to a smaller number having the quality of being most closely related to the number of infections. This was accomplished through a step-wise regression and resulted in the selection of twelve characteristics as being most closely related to the number of infections in a lifetime: marital status, control of temper, spontaneous expression, time lived at a particular address (mobility), self-help, number of jobs held, age at separation of parents, self-esteem, family relations, reaction to infection, educational status, class values.

\section{Results and Conclusions}

(1) No single factor establishes a striking relationship with the number of infections.

(2) A relationship with the number of infections in a lifetime does appear when the factors are considered simultaneously in a multivariate linear regression.

(3) The same factors, even considered simultaneously, are inadequate in establishing a strong relationship with the number of infections in a year or in 3 months. This observation suggests that other factors not measured (e.g. frequency of sexual intercourse) might be involved. It also suggests that the selection of these time periods (a year and 3 months) may have hindered the observation of the relationship between the number of infections and the 33 variates.

(4) When all individuals in the study are given the same average length of time to acquire infections (this is an arbitrary time period determined by the data), a relationship is observed between the number of infections and the interview characteristics.

(5) Twelve of the interview characteristics were observed to have almost the same degree of relationship as did the total 33 characteristics utilized in the partial multiple correlation analysis.

\section{Discussion}

Because gonorrhoea in adults is almost always acquired through intimate sexual contact with an infected person, it is the diseased individual who ultimately controls the effectiveness of any type of public health programme designed to reduce or eradicate gonorrhoea. Where the control programme is based upon case-finding, its success depends upon the patient's supplying information relating to sex contacts. Where the programme is based on education aimed at influencing behaviour which will decrease or eliminate the possibility of infection or re-infection, the patient must take action towards accepting the particular point of view put forth or the programme will fail.

The relationships observed in the study may have implications for educational programmes. For example, it appears that inadequate personal relationships may be an influencing factor. Therefore, if an individual can be helped to gain the skill necessary for developing lasting relationships, one may expect that such change will have an influence towards reducing re-infections with gonorrhoea. If educational programmes could be developed to accomplish these results, it would seem that several points of attack might be feasible. Somehow, before the emergence from adolescence, the individual must be helped to resolve his sex role in terms of ego identity. Without this, it seems that sustained love relationships will be difficult to establish. If ego identity is not established early, re-education of the individual through intensive therapy could possibly accomplish this goal.

A worthwhile effort along these latter lines of thought may involve the exploration of the patient's personality pattern. In this way, patients whose gonorrhoeal infection is symptomatic of a more serious personality disorder could be referred for psychiatric assistance.

The entire question of the repeater must also be considered from quite a different point of view. There are commonly accepted beliefs and levels of behaviour in any society. There are also many variations of behaviour norms approved by subcultures within such societies. Some groups of individuals may adopt and persist in a type of behaviour which is completely acceptable to their immediate associates but which violates the values of the total society. One must consider whether the individuals in this study really have personality problems. From the dominant culture point of view, these people may have aberrant personality characteristics, but from their immediate group's point of view, they may be normal. If this is the situation, attempts to change or alter the personality of such individuals may have a detrimental effect in terms of their relationships within their own group. Rather than re-educate the individual to acquire the skills and ability to form lasting relationships, it might be better to accept the fact that promiscuity is a part of this individual's normal life. Concentration on educational techniques and approaches that would reduce the risk of gonorrhoeal infection from such promiscuity might be indicated. For example, proper utilization of prophylactics might be stressed along with emphasis on more selectivity in choosing sex partners. 


\section{Summary}

Educational programmes, to be successful, must be based upon as complete an understanding as possible of the target group. Efforts tailored to this kind of information will have the best chance of succeeding in changing behaviour. Multivariate analysis may be of value in providing a more complete picture of the individuals for whom an educational programme is contemplated, and should be carefully considered by the health workers concerned with its development.

\section{REFERENCES}

Chapman, J. (1958) Unpublished paper.

Reeder, L. (1961) Unpublished paper.

U.C.L.A. School of Public Health (1963). "Health Districts of the Los Angeles City Health Department", DPHA-2, 61.
Wilner, D. M., Walkley, R., Pinkerton, Ford, and Tayback, M. (1962). "The Housing Environment and Family Life", 1st ed. Johns Hopkins Press, Baltimore.

\section{Une analyse de quelques caractéristiques chez les hommes atteints de blennorragie \\ RésuMé}

Le programme éducatif pour être un succès doit être basé sur une compréhension aussi complète que possible du groupe visé. Les efforts façonnés de ce genre de renseignements auraient la meilleure chance de réussite pour changer le comportement du groupe. L'analyse multiforme peut être d'une valeur en donnant une idée plus complète des individus pour lesquels un programme éducatif est envisagé, et devrait être considérée avec soins par le personnel du service de la santé qui s'intéresse au développement de ce programme. 